\title{
Identification and functional analysis of bacteria in sclerotia of Cordyceps militaris
}

\author{
Li Luo ${ }^{1,2}$, Jiaxi Zhou ${ }^{2}$, Zhongshun Xu ${ }^{2}$, Jingqiang Guan ${ }^{2}$, Yingming Gao ${ }^{2}$, Xiao Zou ${ }^{\text {Corresp. } 1,2}$ \\ 1 Institute of Fungus Resources, Guizhou University, Guiyang, Guizhou, China \\ 2 Department of Ecology, College of Life Science, Guizhou University, Guiyang, Guizhou, China \\ Corresponding Author: Xiao Zou \\ Email address: xzou@gzu.edu.cn
}

Background. Cordyceps militaris is a fungus that parasitizes insects. Compounds from C. militaris are valuable in medicine and functional food. There are many kinds of bacteria in the natural sclerotia of $C$. militaris. However, the community structure of microorganisms in samples from different places may be different, and their corresponding ecological functions require experimental verification.

Methods. We used high-throughput sequencing technology to analyze bacterial 16S rRNA gene sequences in sclerotia of three samples of $C$. militaris from Liaoning Province, China. We isolated, identified and verified the function of culturable bacterial strains from the sclerotia.

Results. Pseudomonas, Pedobacter, Sphingobacterium, and Serratia were the dominant bacterial genera in the sclerotia. And function prediction showed that Pseudomonas and Pedobacter could be heterotrophic, Sphingobacterium could decompose urea, and Serratia could reduce nitrate. Two strains of bacteria isolated from the sclerotia of $C$. militaris, $\mathrm{N}-2$ and $\mathrm{N}-26$, were identified as Stenotrophomonas maltophilia and Pseudomonas baetica, respectively, based on culture and biochemical characteristics. When these isolated strains were co-cultured with $C$. militaris, the mycelium biomass and mycelium pellet diameter decreased, and the content of extracellular polysaccharide increased. Strain N-26 decreased the cordycepin content in C. militaris.

Conclusions. Bacteria in sclerotia have an important effect on the growth of $C$. militaris and the production of its metabolites. 


\section{Identification and functional analysis of bacteria in sclerotia of}

\section{Cordyceps militaris}

3 Li Luo ${ }^{1,2}$, Jiaxi Zhou ${ }^{2}$, Zhongshun $\mathrm{Xu}^{2}$, Jingqiang Guan², Yingming $\mathrm{Gao}^{2}$, Xiao Zou ${ }^{1,2}$

$4 \quad{ }^{1}$ Institute of Fungus Resources, Guizhou University, Guiyang, Guizhou, China

$5 \quad{ }^{2}$ Department of Ecology, College of Life Science, Guizhou University, Guiyang, Guizhou, China

6 Corresponding Author: Xiao Zou

7 West School District of Guizhou University, HangTian Xi Road, Guiyang, Guizhou Province,

$8 \quad 550025$, China

9 Email address: xzou@gzu.edu.cn

10 


\section{Abstract}

12 Background. Cordyceps militaris is a fungus that parasitizes insects. Compounds from $C$. militaris are valuable in medicine and functional food. There are many kinds of bacteria in the natural sclerotia of $C$. militaris. However, the community structure of microorganisms in samples from different places may be different, and their corresponding ecological functions require experimental verification.

Methods. We used high-throughput sequencing technology to analyze bacterial 16S rRNA gene sequences in sclerotia of three samples of $C$. militaris from Liaoning Province, China. We isolated, identified and verified the function of culturable bacterial strains from the sclerotia.

Results. Pseudomonas, Pedobacter, Sphingobacterium, and Serratia were the dominant bacterial genera in the sclerotia. And function prediction showed that Pseudomonas and Pedobacter could be heterotrophic, Sphingobacterium could decompose urea, and Serratia could reduce nitrate. as Stenotrophomonas maltophilia and Pseudomonas baetica, respectively, based on culture and biochemical characteristics. When these isolated strains were co-cultured with $C$. militaris, the mycelium biomass and mycelium pellet diameter decreased, and the content of extracellular polysaccharide increased. Strain N-26 decreased the cordycepin content in C. militaris.

Conclusions. Bacteria in sclerotia have an important effect on the growth of C. militaris and the production of its metabolites.

Keywords: Cordyceps militaris, Microbial community, Co-culture, Functional analysis

\section{Introduction}

Cordyceps militaris is a member of the fungal genus Cordyceps and is used as a traditional

Chinese medicine. It contains a variety of active substances including cordycepin (3'deoxyadenosine), cordyceps polysaccharide, and cordyceps acid (Das et al., 2010). In humans, cordycepin and cordyceps polysaccharide improve immunity (Lee et al., 2020); protect the kidney (Han et al., 2020); have antifatigue (Xu, 2016) and antioxidation properties (Song et al., 2015); inhibit bacterial growth (Ahn et al.,2000), inflammation (Zheng et al.,2020), and tumors 
(Jin et al.,2018); and can be used as an effective anticancer supplement (Tuli et al., 2013). Cordycepin has been suggested for potential use against COVID-19 (Verma, 2020). Much attention has been paid to the cultivation and utilization of C. militaris (Jin et al., 2018; Lee et al., 2020).

C. militaris is a parasitic fungus that can infect larvae, pupae and adults of Lepidoptera, Coleoptera, Diptera and Hymenoptera (Shrestha et al., 2012; Xue et al., 2018); it is distributed throughout the Northern Hemisphere (Zhang et al., 2013). Because the host insects contain a variety of microorganisms, the sclerotia of Cordyceps also contain various microorganisms (Simon et al., 2019). In sclerotia of C. militaris collected in Yunnan Province, China, the bacteria identified included members of the phyla Proteobacteria, Acidobacteria, Bacteroidetes, and Actinobacteria, and the genera Pedobacter, Phyllobacterium, Pseudomonas, Mesorhizobium, Bradyrhizobium, Variovorax, Sphingomonas, and others (Zhang et al., 2021). The bacteria in sclerotia of $C$. sinensis were dominated by Proteobacteria and Actinobacteria and included Pseudomonas, Rhodoferax, Pedobacter, and Sphingomonas (Xia et al., 2016; Xia et al.,2019). In the sclerotia of Cordyceps cicadae, Proteobacteria, Bacteroidetes, and Actinobacteria were the main bacterial groups, and Pseudomonas and Serratia were dominant genera (Mou et al., 2021).

Some of these symbiotic/associated microorganisms have the ability to regulate the growth characteristics and metabolites of Cordyceps. Herbaspirillum and Phyllobacterium on the fruiting body can increase the bioactive compound content of C. militaris (Zhang et al., 2021). Three species of bacteria (Serratia marcescens, Cedecea neteri and Enterobacter aerogenes) isolated from C. cicadae promoted the production of N6-(2-hydroxyethyl) adenosine and decreased the production of adenosine, uridine and guanosine ( $Q u$ et al., 2019). In co-culture, the color of the fungus and the morphology of its mycelia may change (Tauber et al., 2016; Bor et al., 2016). The effect of microorganisms in the sclerotia on the growth of, and metabolite production by, C. militaris needs further research.

In nature, microorganisms coexist in complex communities that interact with each other (Hibbing et al. 2009). These interactions lead to the activation of otherwise silent biosynthetic 
65

66

67

pathways that affect the production of metabolites (Bertrand, et al. 2014). Based on this principle, laboratories sometimes use co-culture to increase the accumulation of metabolites. The purpose of this study was to explore the interaction between the microbes in the sclerotia of wild and cultured C. militaris and the fungus. First, the microbial composition of wild C. militaris harvested in Liaoning Province, China, was analyzed; then, the bacteria were isolated from the sclerotia and identified. These isolated bacteria were then co-cultured with cultured C. militaris to study the effects of the bacteria on the morphology and biomass of mycelia pellets, and the yield of cordycepin and polysaccharide.

\section{Materials and Methods}

\section{Sample preparation}

Wild C. militaris was obtained in October 2019 from 12 insect pupa collected from soil in a broad-leaved mixed forest at an elevation of $240 \mathrm{~m}$ above sea level in Tieling City (42.39 N, 124.26 E), Liaoning Province, China. Cultured C. militaris (L.) Link was stored in the Institute of Fungi Resources of Guizhou University (GZUIFR; strain SYCM1910).

The wild C. militaris obtained from Liaoning Province was divided into three samples for analysis in this work. Sclerotia samples were prepared using the method reported in Zeng, et al., 2019, with slight modifications. The sclerotia formed by C. militaris were rinsed with sterile water to remove residual soil, soaked alternatively with $75 \%$ alcohol and $2 \%$ sodium hypochlorite three times for $20 \mathrm{~s}$ each time, and then rinsed with sterile water. After removal from the body surface of the insect, the sclerotia were used for analysis. Each C. militaris sclerotia sample weighed about $3.5 \mathrm{~g}$. The samples were stored at $-80{ }^{\circ} \mathrm{C}$ until analysis.

\section{Bacterial community determination by culture method}

LB medium contained tryptone $10 \mathrm{~g} / \mathrm{L}$, yeast extract $5 \mathrm{~g} / \mathrm{L}, \mathrm{NaCl} 10 \mathrm{~g} / \mathrm{L}$, and agar $15 \mathrm{~g} / \mathrm{L}(\mathrm{pH} \approx$ 7.0). Potato-dextrose-agar (PDA) contained potato $200 \mathrm{~g} / \mathrm{L}$, glucose $20 \mathrm{~g} / \mathrm{L}$, and agar $15 \mathrm{~g} / \mathrm{L}$ (pH $\approx 7.0$ ). Sabouraud's medium contained glucose $40 \mathrm{~g} / \mathrm{L}$ and peptone $10 \mathrm{~g} / \mathrm{L}(\mathrm{pH} \approx 7.0)$.

Each C. militaris sclerotia sample $(0.5 \mathrm{~g})$ was ground, and its suspension was placed on Luria Bertani agar for microbial isolation at $25^{\circ} \mathrm{C}$. Then, bacteria isolated by the culture method 
92 were observed by scanning electron microscopy (SU8100, Hitachi), and their physiological and biochemical characteristics were identified using bacterial biochemical identification strips HBIG05 and HBIG08 (Qingdao Hopebio Biotechnology Co., Ltd.). Bacterial DNA was extracted according to the procedures for the Bacterial Genomic DNA Extraction Kit DP2002 (Beijing Bioteke Biotechnology Co., Ltd.). The 16S rRNA gene was amplified from all DNA extracts using primers 27F (5'-AGAGTTTGATCCTGGCTCAG-3') and 1492R (5'GGTTACCTTGTTACGACTT-3') (Palkova, et al., 2021). The reaction mixture (25 $\mu \mathrm{L}$ in total) contained $1 \mu \mathrm{mol} / \mathrm{L}$ primers $(1 \mu \mathrm{L}$ each), $10 \mathrm{ng} / \mu \mathrm{L}$ DNA template $(2 \mu \mathrm{L})$, Master Mix (green) (including DNA polymerase, buffer, and dNTPs; $12.5 \mu \mathrm{L}$, product number TSE005, Tsingke Biotechnology Co., Ltd.) and $\mathrm{ddH}_{2} \mathrm{O}(8.5 \mu \mathrm{L})$. The PCR conditions were: predenaturation at 95 ${ }^{\circ} \mathrm{C}$ for $3 \mathrm{~min} ; 32$ cycles of denaturation at $94{ }^{\circ} \mathrm{C}$ for $30 \mathrm{~s}$, annealing at $55{ }^{\circ} \mathrm{C}$ for $30 \mathrm{~s}$, and extension at $72{ }^{\circ} \mathrm{C}$ for $30 \mathrm{~s}$; and a final extension at $72{ }^{\circ} \mathrm{C}$ for $10 \mathrm{~min}$. PCR products were sequenced by Tsingke Biotechnology Co., Ltd. Using the sequence data, BLAST was performed against DNA sequences in GenBank, and the sequences of related species (similarity $>97 \%$ ) were downloaded. Using Pseudomonas carboxydohydrogena as the outgroup, a phylogenetic tree was constructed by the neighbor-joining method using MEGA X software with 1000 bootstrap replicates.

\section{Co-culture of bacteria isolated from sclerotia}

C. militaris SYCM1910 was inoculated on the center of a PDA plate and cultured at $25{ }^{\circ} \mathrm{C}$ for 7 days. Then, bacteria were inoculated at three locations on the periphery of the colony $(25 \mathrm{~mm}$ from the point where $C$. militaris had been inoculated) and culture was continued at $25^{\circ} \mathrm{C}$ for 7 days.

A piece of mycelium of $C$. militaris from a 21-day-old culture on PDA plate culture was inoculated into a 250-mL triangular flask containing $100 \mathrm{~mL}$ Sabouraud's medium and cultured on a magnetic stirrer $(120 \mathrm{rpm})$ at $25^{\circ} \mathrm{C}$ for 3 days. Then, $1 \mathrm{~mL} /$ flask of bacterial suspension (bacterial cell density $1.5 \times 10^{8}$ colony-forming units $/ \mathrm{mL}$ ) was added and culture was continued at $120 \mathrm{rpm}$ and $25{ }^{\circ} \mathrm{C}$ for 7 days. The co-cultured fermentation liquid was filtered using $0.45-\mu \mathrm{m}$ 
119

120

121

122

123

124

125

126

127

128

129

130

131

132

133

134

135

136

137

138

139

140

141

142

143

144

145

and $0.22-\mu \mathrm{m}$ microporous membranes, and then the filtrate was analyzed for the content of cordycepin and extracellular polysaccharides (EPS).

Dry weight of mycelium pellets was determined after the fermentation broth was filtered using qualitative filter paper and the pellets were dried to constant weight at $60{ }^{\circ} \mathrm{C}$. Then, the mycelium pellet diameter was measured using vernier calipers.

Cordycepin content was determined by high-performance liquid chromatography according to the Agricultural Industry Standard NY/T 2116-2012 of the People's Republic of China, using a Thermo Fisher Ultima $3000 \mathrm{RS}$ system and $\mathrm{C}_{18}$ column with mobile phase acetonitrile : water (5:95 v:v) at flow rate $1.0 \mathrm{~mL} / \mathrm{min}$, column temperature $35^{\circ} \mathrm{C}$, detection wavelength $260 \mathrm{~nm}$, and with sample volume $10 \mu \mathrm{L}$. EPS content was determined by the anthrone sulfuric acid method (Guo, et al., 2016).

\section{Bacterial community by non-cultural method}

C. militaris sclerotia samples $(3.0 \mathrm{~g})$ were taken, ground in liquid nitrogen, and total microbial DNA was extracted according to the instructions of the E.Z.N.A.® SOIL DNA Kit (Omega, USA). PCR amplification used TransStart FastPFU DNA Polymerase. The reaction system contained: $5 \times$ FastPFU buffer $(4 \mu \mathrm{L}), 2.5 \mathrm{mmol} / \mathrm{L}$ dNTPs $(2 \mu \mathrm{L}), 5 \mu \mathrm{mol} / \mathrm{L}$ primers $338 \mathrm{~F}\left(5^{\prime}-\right.$ ACTCCTACGGGAGCAG-3') and 806R (5'-GGACTACHVGGGTWTCTA-3') (0.8 $\mu$ L each) targeting the V3-V4 region of 16S rRNA genes (Zeng \& An, 2021), FastPFU Polymerase (0.4 $\mu \mathrm{L})$, bovine serum albumin $(0.2 \mu \mathrm{L}, 1 \mu \mathrm{g} / \mu \mathrm{L})$, and template DNA (10 ng), supplemented with $\mathrm{ddH}_{2} \mathrm{O}$ to $20 \mu \mathrm{L}$. An ABI Gene AMP® 9700 PCR instrument was used for the reaction. The reaction parameters were $95^{\circ} \mathrm{C}$ for $3 \mathrm{~min} ; 30$ cycles of $95^{\circ} \mathrm{C}$ for $30 \mathrm{~s}, 55^{\circ} \mathrm{C}$ for $30 \mathrm{~s}$, and $72^{\circ} \mathrm{C}$ for $45 \mathrm{~s}$; and a final extension at $72{ }^{\circ} \mathrm{C}$ for $10 \mathrm{~min}$. The amplified products were sent to Shanghai Major Biomedical Technology Co., Ltd. and sequenced using the Illumina MiSeq platform. Paired-end reads obtained by MiSeq sequencing were first stitched by overlap, and sequence quality was controlled and filtered at the same time. Effective sequences were obtained by distinguishing samples according to barcode and primer sequences at both ends of the sequence, and sequence direction was corrected to obtain optimized sequences. Using UPARSE software 
146 (http://www.drive5.com/uparse/), repetitive sequence operational taxonomic unit (OTU)

147 clustering was carried out with a threshold of $97 \%$ similarity, chimeras were removed in the

148 process of clustering, and the RDP database (http://rdp.cme.msu.edu/) was used for OTU 149 annotation.

150 The raw sequence reads obtained in this study were deposited in the NCBI Sequence Read 151 Archive database under accession number PRJNA722375. FAPROTAX

152 (http://www.zoology.ubc.ca/louca/FAPROTAX/), a tool that can predict ecological functions of 153 bacterial and archaea taxa from 16S rRNA amplicon sequencing (Sansupa, et al., 2021), was

154

155 156 157

used to identify ecological functions of OTUs. An OTU abundance table and taxonomic annotation of OTUs were inputted in the corresponding option box, and the PLOT option was selected. Then, predicted function output was obtained as an Excel spreadsheet. A heatmap was generated using the online tool at the website www.ehbio.com/ImageGP/.

\section{Data analysis}

Statistical analysis of the experimental data was performed using SPSS software v.22.0. The least significant difference test was used for one-way analysis of variance.

\section{Results}

\section{Isolation and identification of bacteria}

Two pure strains of bacteria, N-2 and N-26, were isolated from sclerotia of C. militaris. Strain N2 is short rod-shaped $(7.2-9.4 \times 3.2-3.8 \mu \mathrm{m})($ Fig. 1) and Gram-negative; colonies were slightly convex, pale yellow, smooth, moist, and opaque. Physiological and biochemical tests (Table S1) showed that strain N-2 is motile, can decompose glucose to produce pyruvate, and can decarboxylate the pyruvate and convert it into alcohol and other substances. In addition, ornithine decarboxylase, lysine decarboxylase and amino acid decarboxylase were detected, indicating that strain $\mathrm{N}-2$ can decarboxylate amino acids $(-\mathrm{COOH})$ to produce an amine and $\mathrm{CO}_{2}$. Strain N-2 cannot use mannitol, inositol, sorbitol, melibiose, ribitol, raffinose, xylose, or maltose as carbon sources. Using the methods described in the eighth edition of "Bergey's Manual of Systematic Bacteriology," strain N-2 was identified as belonging to the genus Stenotrophomonas. 
173

174

175

176

177

178

179

180

181

182

183

184

185

186

187

188

189

190

191

192

193

194

195

196

197

198

199

By BLAST analysis, the 16S rRNA gene sequence of strain N-2 was found to be $99.93 \%$ identical to that of S. maltophilia GZUIFR-YC01. Strain N-2 was identified as S. maltophilia (Hugh) (Fig. S1) (NCBI accession number: MW829549).

Strain N-26 is short rod-shaped $(9.5-11.5 \times 4.2-5 \mu \mathrm{m})$, Gram-negative, and its colonies are yellow, smooth, moist and opaque, with a central bulge. The semi-solid agar (dynamic test) of strain N-26 was positive, the Voges-Proskauer test was positive, and the Methyl Red test was negative. The strain was positive for ornithine decarboxylase, lysine decarboxylase and amino acid decarboxylase. The strain could not use mannitol, inositol, sorbitol, melibiose alcohol, raffinose, xylose, or maltose as carbon sources. In BLAST analysis, the 16S rRNA gene sequence of strain N-26 was 99.71\% identical to that of Pseudomonas baetica YHNG5 (Fig. S1), which led to the identification of strain N-26 as P. baetica (Lopez) (NCBI Accession number: MW829550).

\section{Interaction between isolated bacteria and $C$. militaris}

On PDA plates, $S$. maltophilia $\mathrm{N}-2$ had an inhibitory effect on the growth of mycelium of $C$. militaris. Strain N-2 released something that slowed the growth of $C$. militaris mycelia near the area of S. maltophilia N-2 growth (Fig. 2a). P. baetica N-26 did not inhibit mycelial growth on PDA plates (Fig. 2b).

The dry weight of mycelium pellets decreased from $1.57 \mathrm{~g} /$ flask in the control to $0.21 \mathrm{~g} / \mathrm{flask}$ in the presence of $S$. maltophilia strain $\mathrm{N}-2$ or $0.35 \mathrm{~g} /$ flask in the presence of $P$. baetica strain N26 strain after co-culture with $C$. militaris for 7 days; these differences were highly significant when compared with the control $(\mathrm{N}-2: F=885.476, P<0.001 ; \mathrm{N}-26: F=493.275, P<0.001)$ (Fig. 3A). The diameter of mycelium pellets decreased from $7.38 \mathrm{~mm}$ in the control to 3.29 and $3.63 \mathrm{~mm}$ after culture in the presence of strains $\mathrm{N}-2$ and $\mathrm{N}-26$, respectively $(\mathrm{N}-2: F=1240.221$, $P<0.01 ; \mathrm{N}-26: F=605.933, P<0.001)$ (Fig. 3B). The addition of strain N-26 significantly decreased the cordycepin content of the culture medium from $3015.73 \mu \mathrm{g} / \mathrm{g}$ in the control to $2537.77 \mu \mathrm{g} / \mathrm{g}(F=22.501, P=0.009)$. However, addition of strain N-2 had little effect (3102.00 $\mu \mathrm{g} / \mathrm{g} ; F=0.285, P=0.622$ ) (Fig. 3C). The EPS content was increased after bacteria were added 
200

201

202

203

204

205

206

207

208

209

210

211

212

213

214

215

216

217

218

219

220

221

222

223

224

225

226

to $C$. militaris culture, and the difference was significant compared with the control (N-2: 481.43 $\mathrm{mg} / \mathrm{g}, F=291.121, P<0.001 ; \mathrm{N}-26: 326.87 \mathrm{mg} / \mathrm{g}, F=93.546, P=0.001$; control: $86.20 \mathrm{mg} / \mathrm{g}$ ) (Fig. 3D).

\section{Bacterial community composition and ecological function}

A total of 204,067 valid sequences were detected in three sclerotia samples of C. militaris collected in October 2019 in Liaoning Province, China; 62,929-71,212 sequences were obtained for each sample, with average length $423.45-425.61 \mathrm{bp}$. With the increase of the number of sample sequences, the Shannon-Winner index curve flattened out (Fig. S2), indicating that the sequencing data depth in this experiment comprehensively reflected the bacterial community in the samples.

Bacteria identified in the sclerotia included 21 phyla, 46 classes, 123 orders, 195 families, 321 genera, 450 species, and 549 operational taxonomic units (OTUs). At the phylum level (Fig. 4), Proteobacteria (average relative abundance of OTU 68\%), Bacteroidetes (24\%), and Actinobacteria (8\%) were dominant. At the genus level, Pseudomonas (17\%), Unclassified Enterobacteriaceae (14\%), Pedobacter (11\%), Sphingobacterium (11\%), Serratia (10\%), Rhodococcus (6\%), and Acromobacter (6\%) were dominant. Forty-two OTUs were common to the three samples (Fig. S3), accounting for only $7.65 \%$ of the total number of OTUs. These 42 OTUs were uploaded to the FAPROTAX system for analysis, and predicted functions of 17 genera represented by 21 OTUs were identified (Fig. 5; Table S2). OTU1490 (Stenotrophomonas) is animal parasitic or symbiotic, and a human pathogen; it actively participates in the nitrogen cycle. OTU2342 (Sphingobacterium) is involved in urea decomposition. OTUs 1448, 2330 and 2314 (Pseudomonas) are chemoheterotrophic. OTUs 1539 and 1423 (Rhodococcus) degrade aromatic hydrocarbons and aliphatic non-paraffin hydrocarbons.

\section{Discussion}

In our study, we isolated two bacteria belonging to the microbiota of $C$. militaris sclerotia, $S$. maltophilia $\mathrm{N}-2$ and P. baetica $\mathrm{N}-26$. Then, we co-cultured these bacteria with $C$. militaris, and 
227 found that both of them increased the EPS content of C. militaris, but Pseudomonas baetica N-

22826 decreased the cordycepin content.

229 The bacteria present in sclerotia of C. militaris sampled from Liaoning Province, China, were

230 identified using high-throughput sequencing technology. Pseudomonas were more abundant in

231 sclerotia of C. militaris isolated in Liaoning Province than in C. militaris isolated in Yunnan

232 Province, while Phyllobacterium, Mesorhizobium, and Bradyrhizobium were less abundant in the

233 former (Zhang et al., 2021). The relative abundance of Mesorhizobium, Bradyrhizobium,

234 Sphingomonas, and Labrys in sclerotia samples from Liaoning Province was lower than in

235 samples from Yunnan Province (Table 1). Pseudomonas, Pedobacter, Phyllobacterium,

236 Mesorhizobium, Bradyrhizobium, Sphingomonas, Variovorax, and Labrys were found in C.

237 militaris samples from both Yunnan Province (southwest China, 25.40 N, 102.92 E) and

238 Liaoning Province (northeast China, 42.39 N, 124.26 E), but their relative abundances were

239 different, which may be related to differences of the insect host and environmental conditions

240 (Yun et al., 2014). The bacteria found in the sclerotia may be key microorganisms in the

241 microenvironment of C. militaris and perform important functions (Lemanceau et al., 2017).

242 Functional prediction (Table 1) showed that Pseudomonas, Pedobacter, Labrys, and

243 Sphingomonas are chemoheterotrophic, while Stenotrophomonas functions in the nitrogen cycle.

244 Pseudomonas, Phyllobacterium, Mesorhizobium, Bradyrhizobium, Pedobacter, Variovorax, and

245 Sphingomonas belong to the microbiome of the plant rhizosphere (Etesami \& Glick, 2020; Yin et

246 al., 2020). These microorganisms may help to maintain plant hormone balance, control root

247 development, promote nutrient acquisition, prevent disease, improve plant growth, and maintain

248 plant health (Xu et al., 2018). Such microorganisms are also ingested by root-feeding insects.

249 When Cordyceps spp. invade insects, the microorganisms in the insect gut interact with the

250 fungus (Lei et al.,2015; Zhong et al., 2014).

251 With regard to the potential ecological functions of the two strains we isolated,

252 Stenotrophomonas maltophilia, a parasitic bacterium of insects (Gandotra et al., 2018), can

253 promote the digestion and absorption of food by the host by secreting enzymes such as cellulase, 
254

255

256

257

258

259

260

261

262

263

264

265

266

267

268

269

270

271

272

273

274

275

276

277

278

279

280

amylase, protease, and chitinase. These enzymes inhibit the integrity of fungal hyphae and biofilm formation (Ali, et al.,2019; Jankiewicz \& Brzezinska, 2015; Rossi et al.,2014). In insects, S. maltophilia can inhibit the growth of Beauveria bassiana (a fungus that parasitizes arthropods) (Zhou et al., 2018). Therefore, S. maltophilia can play a protective role in an insect host. S. maltophilia participates in the sulfur and nitrogen cycles, degrades complex compounds and pollutants, and promotes plant growth and health (An \& Berg, 2018). We conclude that $S$. maltophilia plays an important ecological role in the sclerotia of C. militaris.

Pseudomonas has many functions, e.g., P. fluorescens secretes luciferin and inhibits the growth of Escherichia coli in insects (Roberts et al., 2018), decomposes wood, synthesizes multiple vitamins, and suppresses fungi in beetles (Peral-Aranega et al.,2020). P. aeruginosa strain BGF-2 isolated from German cockroach could inhibit the growth of B. bassiana (Huang et al., 2013). Pseudomonas has a flexible metabolism that allows it to synthesize a wide range of antibiotics to ward off competitors, protect itself from predators, and produce chemical signaling molecules that sustain intraspecies and interspecies interactions (G"otze \& Stallforth, 2019).

The relative abundance of bacteria in insects may be related to growth stimulation by parasitic fungi. For example, the biomass of P. fragi (a bacterium found in Thitarodes and Hepialus ghost moths) increased after invasion by $C$. sinensis, and $P$. fragi became the dominant bacterium and participated in the process of larval mummification (Wu et al., 2020). Both Stenotrophomonas and Pseudomonas have been reported to inhibit conidial germination and mycelial growth of $B$. bassiana (Zhou et al., 2018). The two bacterial strains tested in this study had a similar effect on the hyphae of $C$. militaris. These findings indicate that the use of parasitic bacteria to inhibit fungal invasion is a protective mechanism of insects.

In the present study, the biomass of C. militaris decreased after co-culture with strain N-2 ( $S$. maltophilia) or $\mathrm{N}-26$ (P. baetica), so the cordycepin content in the culture medium decreased accordingly. Several mechanisms could explain this effect. One is inhibition of the expression of cordycepin-synthesis-related genes (cns1, cns2, cns3, and cns4) (Zheng et al., 2011); this can be verified by quantitative PCR in later study. Alternatively, (a) key enzyme(s) involved in 
281

282

283

284

285

286

287

cordycepin synthesis may have been inhibited. It is also possible that the mycelium structure was damaged in the co-culture process, which could be observed by using cryo-electron microscopy after co-culture. Alternatively, cordycepin may be produced at around the usual level but used by the co-cultured bacterium.

Because cordycepin has antibacterial function (Jiang et al., 2019), we speculate that its ecological role is to inhibit the growth of some bacteria, to create good conditions for C. militaris and keep the insect host from decaying. Our data indicate that some symbiotic bacteria may inhibit the production of cordycepin. We speculate that other symbiotic bacteria may promote the production of cordycepin. Thus, we aim to isolate other microorganisms from the sclerotia and further explore the relationships between the isolates and C. militaris. When increasing numbers of interactions are revealed, the ecological functions of microbes in the sclerotia will become clearer.

The biosynthetic potential of many bacterial and fungal strains is much greater than previously thought. For example, in Pseudoalteromonas sp. MEBiC 03485 co-cultured with Porphyridium cruentum UTEX 161, the content of sulfated polysaccharide was increased (Han et al., 2020). The mechanism may lie in the effect of some small-molecule elicitors on the related transcription of secondary metabolite gene clusters (Pettit, 2011). The results of a previous study suggest that the increased production of EPS in the present study may be related to the protein phosphoglucomutase (Wang et al., 2021). However, it is unclear whether the increase was in the original type of polysaccharide or in new types of polysaccharide; this requires further study.

\section{Conclusion}

The microbiota of the sclerotia of $C$. militaris contains a diversity of bacteria, among which Pseudomonas, Pedobacter, and Serratia are the dominant genera. This study reveals the interactions between $C$. militaris and isolated strains of $S$. maltophilia and P. baetica; these bacteria had inhibitory effects on the biomass and mycelial pellet diameter of $C$. militaris, and increased its EPS content. Furthermore, P. baetica strain N-26 decreased the cordycepin content in C. militaris. These results enrich the study of microbial interactions in entomogenous fungal 
308

309

310

311

312

313

314

315

316

317

318

319

320

321

322

323

324

325

326

327

328

329

330

331

332

333

334

335

336

337

338

339

340

microenvironments and provide reference for improving the use of metabolites.

\section{Acknowledgments}

We thank Chun-Bo Dong for some suggestion of the manuscript.

\section{Reference}

Ahn YJ, Park SJ, Lee SG, Shin SC, Choi, DH. 2000. Cordycepin: Selective growth inhibitor derived from liquid culture of Cordyceps militaris against Clostridium spp. Journal of Agricultural and Food Chemistry 48(7): 2744-2748 DOI 10.1021/jf990862n.

Ali Huda RK, Hemeda NF, Abdelaliem YF. 2019. Symbiotic cellulolytic bacteria from the gut of the subterranean termite psammotermes hypostoma Desneux and their role in cellulose digestion. AMB Express 9:111-120 DOI 10.1186/s13568-019-0830-5.

An SQ, Berg G. 2018. Stenotrophomonas maltophilia. Trends in Microbiology 26: 637-638 DOI 10.1016/j.tim.2018.04.006.

Asaf S, Numan M, Khan AL, Al-Harrasi A. 2020. Sphingomonas: from diversity and genomics to functional role in environmental remediation and plant growth. Critical Reviews in Biotechnology 40(2):1-15 DOI 10.1080/07388551.2019.1709793.

Bor B, Cen LJ, Agnello M, Shi WY, He XS. 2016. Morphological and physiological changes induced by contact-dependent interaction between Candida albicans and Fusobacterium nucleatum. Scientific Reports 6:27956 DOI 10.1038/srep27956.

Das Shonkor K, Masuda M, Sakurai A, Sakakibara M. 2010. Medicinal uses of the mushroom Cordyceps militaris: current state and prospects. Fitoterapia 81(8):961-968 DOI 10.1016/j.fitote.2010.07.010.

Etesami H, Glick BR. 2020. Halotolerant plant growth-promoting bacteria: Prospects for alleviating salinity stress in plants. Environmental and Experimental Botany 178:104124. DOI 10.1016/j.envexpbot.2020.104124.

Gandotra S, Kumar A, Naga K, Bhuyan PM, Gogoi DK, Sharma K, Subramanian S. 2018. Bacterial community structure and diversity in the gut of muga silkworm, Antheraea assamensis (lepidoptera: saturniidae) from india. Insect Molecular Biology 27(5):603-619 DOI 10.1111/imb.12495.

Götze S, Stallforth P. 2019. Structure, properties, and biological functions of nonribosomal lipopeptides from Pseudomonads. Natural Product Reports 37(1):29-54 DOI 10.1039/C9NP00022D.

Guo M, Guo S, Yang H, Bu N, Dong C.2016. Comparison of Major Bioactive Compounds of the Caterpillar Medicinal Mushroom, Cordyceps militaris (Ascomycetes), Fruiting Bodies 
341

342

343

344

345

346

347

348

349

350

351

352

353

354

355

356

357

358

359

360

361

362

363

364

365

366

Cultured on Wheat Substrate and Pupae. International Journal of Medicinal Mushrooms 18(4):327-336 DOI 10.1615/IntJMedMushrooms.v18.i4.60.

\section{Han F, Dou M, Wang YX, Xu CX, Li Y, Ding XM, Xue WJ, Zheng J, Tian PX, Ding CG.} 2020. Cordycepin protects renal ischemia/reperfusion injury through regulating inflammation, apoptosis, and oxidative stress. Acta Biochim Biophys Sin 52(2):125-132 DOI 10.1093/abbs/gmz145.

Han SI, Jeon MS, Heo YM, Kim S, Choi YE. 2019. Effect of Pseudoalteromonas sp. MEBiC 03485 on biomass production and sulfated polysaccharide biosynthesis in Porphyridium cruentum UTEX 161.2020. Bioresource Technology 302: 1-9 DOI 10.1016/j.biortech.2020.122791.

Huang YH, Wang XJ, Zhang F, Huo XB, Fu RS, Liu JJ, Sun WB, Kang DM, Jing X. 2013. The identification of a bacterial strain BGI1 isolated from the intestinal flora of Blattella germanica, and its anti-entomopathogenic fungi activity. Journal of Economic Entomology 106(1):43-49 DOI 10.1603/EC12120.

Jankiewicz U, Brzezinska MS. 2015. Purification, characterization, and gene cloning of a chitinase from Stenotrophomonas maltophilia N4. Journal of Basic Microbiology 55(6):929929 DOI 10.1002/jobm.201400717.

Jiang Q, Lou ZX, Wang HX, Chen C. 2019. Antimicrobial effect and proposed action mechanism of cordycepin against Escherichia coli and Bacillus subtilis. Journal of Microbiology 57(4): 288-297 DOI 10.1007/s12275-019-8113-z.

Jin Y, Meng X, Qiu ZD, Su YP, Yu P, Qu P. 2018. Anti-tumor and anti-metastatic roles of cordycepin, one bioactive compound of Cordyceps militaris. Saudi Journal of Biological Sciences 25(5):991-995 DOI 10.1016/j.sjbs.2018.05.016.

Lee CT, Huang KS, Shaw JF, Chen JR, Kuo WS, Shen GX, Grumezescu AM, Holban AM, Wang YT, Wang JS, Hsiang YP, Lin YM, Hsu HH, Yang CH. 2020.Trends in the immunomodulatory effects of Cordyceps militaris: total extracts, polysaccharides and cordycepin. Frontiers in Pharmacology 11:1-20 DOI 10.3389/fphar.2020.575704.

Lei W, Zhang GR, Peng QY, Liu X. 2015. Development of Ophiocordyceps sinensis through Plant-Mediated Interkingdom Host Colonization. International Journal of Molecular Sciences 16(8): 17482-17493 DOI 10.3390/ijms160817482.

Lemanceau P, Blouin M, Muller D, Moënne-Loccoz Y. 2017. Let the Core Microbiota Be Functional. Trends in Plant Science 22(7):583-595 DOI 10.1016/j.tplants.2017.04.008.

Margesin R, Shivaji S. 2011. Pedobacter. Bergey's Manual of Systematics of Archaea and Bacteria, Online(C2015 Bergey’s Manual Trust DOI 10.1002/9781118960608.gbm00360. 
375

376

377

378

379

380

381

382

383

384

385

386

387

388

389

390

391

392

393

394

395

396

397

398

399

400

401

402

403

404

405

406

407

408

409

Mou D, Zeng ZY, Zhong WL, Zhou JX, Qu JJ, Zou X. 2021. Bacterial community structure and function of Cordyceps cicadae microecosystem in Guiyang. Acta Microbiologica Sinica 61(2):469-481 DOI 10.13343/j.cnki.wsxb.20200540.

Palkova L, Tomova A, Repiska G, Babinska K, Bokor B, Mikula I, Minarik G, Ostatnikova D, Soltys K. 2021. Evaluation of 16S rRNA primer sets for characterisation of microbiota in paediatric patients with autism spectrum disorder. Scientific Reports 11(1): 6781. DOI 10.1038/s41598-021-86378-w.

Peral-Aranega E, Saati-Santamaría Z, Kola`rik M, Rivas R, García-Fraile P. 2020. Bacteria belonging to Pseudomonas typographi sp. nov. from the Bark beetle Ips typographus Have Genomic Potential to Aid in the Host Ecology. Insects 11(9): 593 DOI 10.3390/insects11090593.

Pettit R. 2011. Small-molecule elicitation of microbial secondary metabolites. Microbial Biotechnology 4(4): 471-478. DOI 10.1111/j.1751-7915.2010.00196.x

Qu QS., Yang F, Zhao CY, Shi XY. 2019. Analysis of the bacteria community in wild cicadae and its influence on the production of HEA and nucleosides in Cordyceps cicadae. Journal of Applied Microbiology 127(6):1759-1767 DOI 10.1111/jam.14432.

Roberts DC, Fleischer SJ, Sakamoto JM, Rasgon JL. 2018. Potential biological control of Erwinia tracheiphila by internal alimentary canal interactions in Acalymma vittatum with Pseudomonas fluorescens. Journal of Applied Microbiology 125(4):1137-1146 DOI 10.1111/jam.13950.

Rossi BP, García C, Alcaraz E, Franco M. 2014. Stenotrophomonas maltophilia interferes via the DSF-mediated quorum sensing system with Candida albicans filamentation and its planktonic and biofilm modes of growth. Revista Argentina De Microbiología 46(4):288-297 DOI 10.1016/S0325-7541(14)70084-7.

Sansupa C, Mohamed WSF, Hossen S, Disayathanoowat T, Wubet T, Purahong W. 2021. Can We Use Functional Annotation of Prokaryotic Taxa (FAPROTAX) to Assign the Ecological Functions of Soil Bacteria?. Applied Sciences 11(2):688 DOI 10.3390/app11020688.

Shrestha B, Zhang WM, Zhang YJ, Liu XZ. 2012. The medicinal fungus Cordyceps militaris: research and development. Mycological Progress 11(3):599-614 DOI 10.1007/s11557-0120825-y.

Simon JC, Marchesi Julian R, Mougel C, Selosse MA. 2019. Host-microbiota interactions: from holobiont theory to analysis. Microbiome 7:5 DOI 10.1186/s40168-019-0619-4.

Song JJ, Wang YW, Teng MY, Cai GS, Xu HK, Guo HX, Liu Y, Wang D, Teng LS. 2015. Studies on the Antifatigue Activities of Cordyceps militaris Fruit Body Extract in Mouse 
Model. Evidence-Based Complementray and Alternative Medicine 2015:174616 DOI $10.1155 / 2015 / 174616$.

Tauber JP, Schroeckh V, Shelest E, Brakhage AA, HoffmeisterD. 2016. Bacteria induce pigment formation in the basidiomycete Serpula lacrymans. Environmental Microbiology 18(12):5218-5227 DOI 10.1111/1462-2920.13558.

Tuli Hardeep S, Sharma Anil K, Sandhu Sardul S, Kashyap D. 2013. Cordycepin: A bioactive metabolite with therapeutic potential. Life Science 93(23):863-869 DOI 10.1016/j.lfs.2013.09.030.

Verma Akalesh K. 2020. Cordycepin: a bioactive metabolite of Cordyceps militaris and polyadenylation inhibitor with therapeutic potential against COVID-19. Journal of Biomolecular Structure \& Dynamics 3:1-8 DOI 10.1080/07391102.2020.1850352.

Wu H, Rao ZC, Cao L, Clercq PD, Han RC. 2020. Infection of Ophiocordyceps sinensis fungus causes dramatic changes in the microbiota of its Thitarodes host. Frontiers in Microbiology 11:577268 DOI 10.3389/fmicb.2020.577268.

Xia F, Liu Y, Guo MY, Shen GR, Lin J, Zhou XW. 2016. Pyrosequencing analysis revealed complex endogenetic microorganism community from natural DongChong XiaCao and its microhabitat. BMC Microbiology 16:196 DOI 10.1186/s12866-016-0813-5.

Xia F, Zhou X, Liu Y, Li YL, Bai XH, Zhou XW. 2019. Composition and predictive functional analysis of bacterial communities inhabiting Chinese cordyceps insight into conserved core microbiome. BMC Microbiology 19:105 DOI 10.1186/s12866-019-1472-0.

Xu J, Zhang YZ, Zhang PF, Trivedi P, Riera N, Wang YY, Liu X, Fan GY, Tang JL, Coletta-Filho HD, Cubero J, Deng XL, Ancona V, Lu Z, Roper MC, Capote N, Catara V, Pietersen G, Verniere C, AI-Sadi AM, Li L, Yang F, Xu X, Wang J, Yang HM, Jin T, Wang N.2018. The structure and function of the global citrus rhizosphere microbiome. Nature Communications 9:4984

DOI 10.1038/s41467-018-07343-2.

Xu YF. 2016. Effect of Polysaccharide from Cordyceps militaris (Ascomycetes) on Physical Fatigue Induced by Forced Swimming. International Journal of Medicinal Mushrooms 18(12):1083-1092 DOI 10.1615/IntJMedMushrooms.v18.i12.30.

Xue YF, Wu LL, Ding YL, Cui XM, Han ZZ, Xu H. 2018. A new nucleoside and two new pyrrole alkaloid derivatives from Cordyceps militaris. Natural Product Research 34(3):1-10 DOI 10.1080/14786419.2018.1531861.

Yin C, Vargas JMC, Schlatter DC, Hagerty CH, Hulbert SH, Paulitz TC. 2020. Rhizosphere community selection reveals bacteria associated with reduced root disease. Microbiome 9(1):86 DOI 10.1186/s40168-020-00997-5. 
Yin DL., Wang RL., Duan HB. 2014. Optimization of extraction technology of polysaccharide from Cordyceps militaris fruiting body. Jiangsu Agricultural Sciences 42(7):277-279 DOI 10.15889/j.issn.1002-1302.2014.07.262.

Yun JH, Roh SW, Whon TW, Jung MJ, Kim MS, Park DS, Yoon C, Nam YD, Kim YJ, Choi JH, Kim JY, Shin NR, Kim SH, Lee WJ, Bae JW. 2014. Insect gut bacterial diversity determined by environmental habitat, diet, developmental stage, and phylogeny of host. Applied and Environmental Microbiology 80(17): 5254-5264 DOI 10.1128/AEM.01226-14.

Zeng Q, An S. 2021. Identifying the Biogeographic Patterns of Rare and Abundant Bacterial Communities Using Different Primer Sets on the Loess Plateau. Microorganisms 9(1):139 DOI 10.3390/microorganisms9010139.

Zeng ZY, Xu ZS, Mou D., Xu SH., Du F, Zhou JX, Zou X. 2019. Analyses on the structure and ecological function of fungal community in sclerotia, external mycelial layer covering ossified cicada epidermis and habitat soil of Isaria cicadae. Mycosystema 38(10):1710-1723 DOI 10.13346/j.mycosystema.190138.

Zhang S., Zhang YJ., Shrestha B. Xu JP, Wang CS, Liu XZ. 2013. Ophiocordyceps sinensis and Cordyceps militaris: research advances, issues and perspectives. Mycosystema 32(4):577597 DOI 10.13346/j.mycosystema.2013.04.001.

Zhang XM, Tang DX, li QQ, Wang YB, Xu ZH, Li WJ, Yu H. 2021. Complex microbial communities inhabiting natural Cordyceps militaris and the habitat soil and their predicted functions. Antonie van Leeuwenhoek 114(4):465-477 DOI 10.1007/s10482-021-01534-6.

Zheng P, Xia YL, Xiao GH, Xiong CH, Hu X, Zhang SW, Zheng HJ, Huang Y, Zhou Y, Wang SY, Zhao GP, Liu XZ, Wang CS. 2011. Genome sequence of the insect pathogenic fungus Cordyceps militaris, a valued traditional Chinese medicine. Genome Biology. 12(1): R116 DOI 10.1186/gb-2011-12-11-r116.

Zheng Y, Li L, T TC. 2020. Cordyceps polysaccharide ameliorates airway inflammation in an ovalbumin-induced mouse model of asthma via TGF- $\beta 1 /$ Smad signaling pathway. Respiratory Physiology \& Neurobiology 276: 103412 DOI 10.1016/j.resp.2020.103412.

Zhong X, Peng QY, Li SS, Chen H, Sun HX, Zhang RG, Liu X. 2014. Detection of Ophiocordyceps sinensis in the roots of plants in alpine meadows by nested-touchdown polymerase chain reaction. Fungal Biology 118(4): 359-363 DOI 10.1016/j.funbio.2013.12.005.

Zhou FY, Wu XQ, Xu LT, Guo SH, Chen GH, Zhang XJ. 2018. Repressed Beauveria bassiana infections in Delia antiqua due to associated microbiota. Pest Management Science 75(1):170-179 DOI 10.1002/ps.5084. 


\section{List of Figures and Tables}

482 Figure 1 Scanning electron micrographs of strains.

483 Figure 2 Co-culture on potato-dextrose-agar.

484 Figure 3 Effects of coculture of isolated bacterial strains with C. militaris.

485 Figure 4 Taxonomic composition of the microbiome in sclerotia of Cordyceps militaris.

486 Figure 5 Functional prediction of the bacterial core microbiome in sclerotia of $C$. militaris using 487 FAPROTAX.

488 Table 1 Relative abundance and function prediction of bacterial composition in sclerotia of $C$. 489 militaris isolates.

490

491 Supplementary Information

492 Supplemental Figure S1. Phylogenetic tree of the strains.

493 Supplemental Figure S2. The Shannon rarefaction curve of bacterial community on OTU.

494 Supplemental Figure S3. Venn of three samples on OTU.

495 Supplemental Table S1. Some physiological and biochemical characteristics of the N-2 and N49626 strains.

497 Supplemental Table S2. Classification, functional prediction and abundance of OTU in 498 sclerotinia of $C$. militaris.

499

500 Abbreviations

501 C. militaris: Cordyceps militaris;

502 S. maltophilia: Stenotrophomonas maltophilia;

503 P. baetica: Pseudomonas baetica;

504 O. sinensis: Ophiocordyceps sinensis;

505 VP test: Voges-Proskauer test;

506 MR test: Methyl rea test;

507 BLAST: Basic local alignment search tool; 
508 NCBI: National center for biotechnology information;

509 HPLC: High performance liquid chromatography;

510 HEA: N6-(2-hydroxyethyl)-adenosine;

511 SE: Scan Electron microscopic;

512 EPS: Extracellular polysaccharide;

$513 \mathrm{ddH}_{2} \mathrm{O}$ : Double distilled water;

514 LSD: Least Significant Difference;

515 ANOVA: Analysis of Variance; 
Figure 1

Scanning electron micrographs of strains.

(A) N-2 and (B) N-26. Note: In Fig. 1a, the reticular-like structure on the surface of the bacteria is secretion by the bacteria.

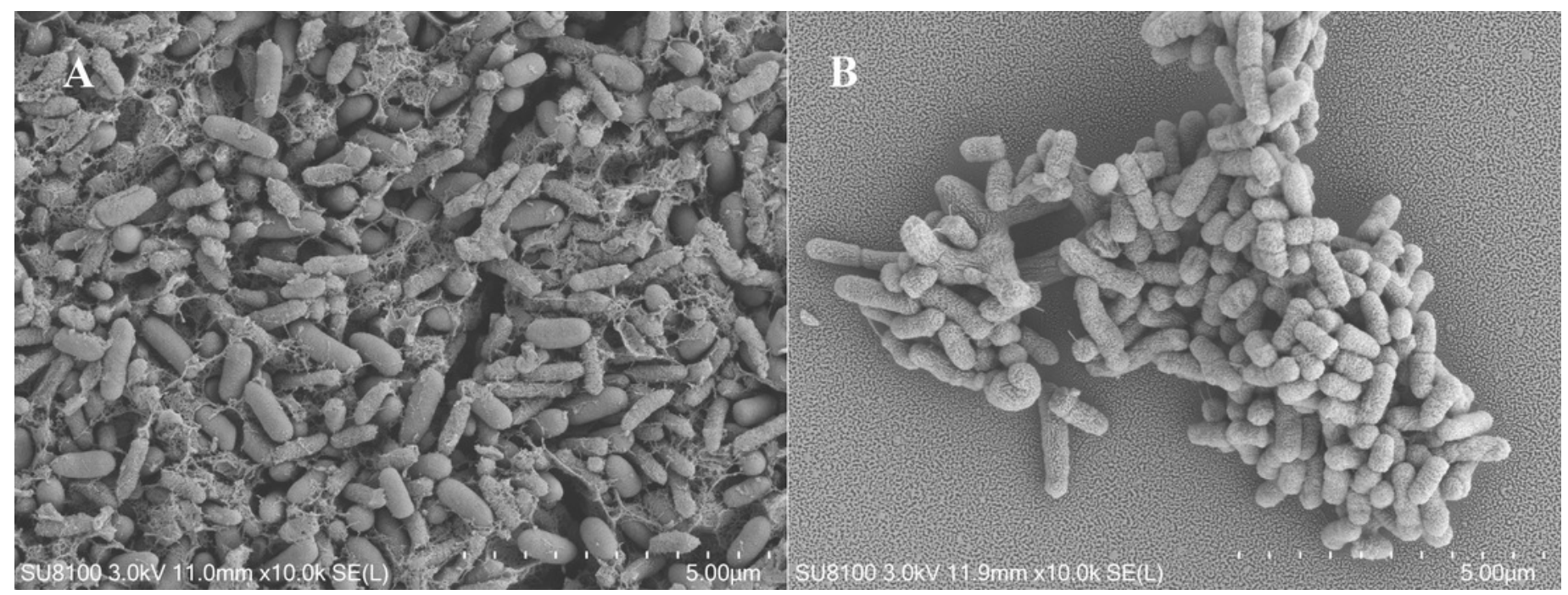




\section{Figure 2}

Co-culture on PDA plates.

(A) Strain N-2 and C. militaris, (B) strain N-26 and C. militaris, (C) C. militaris only.

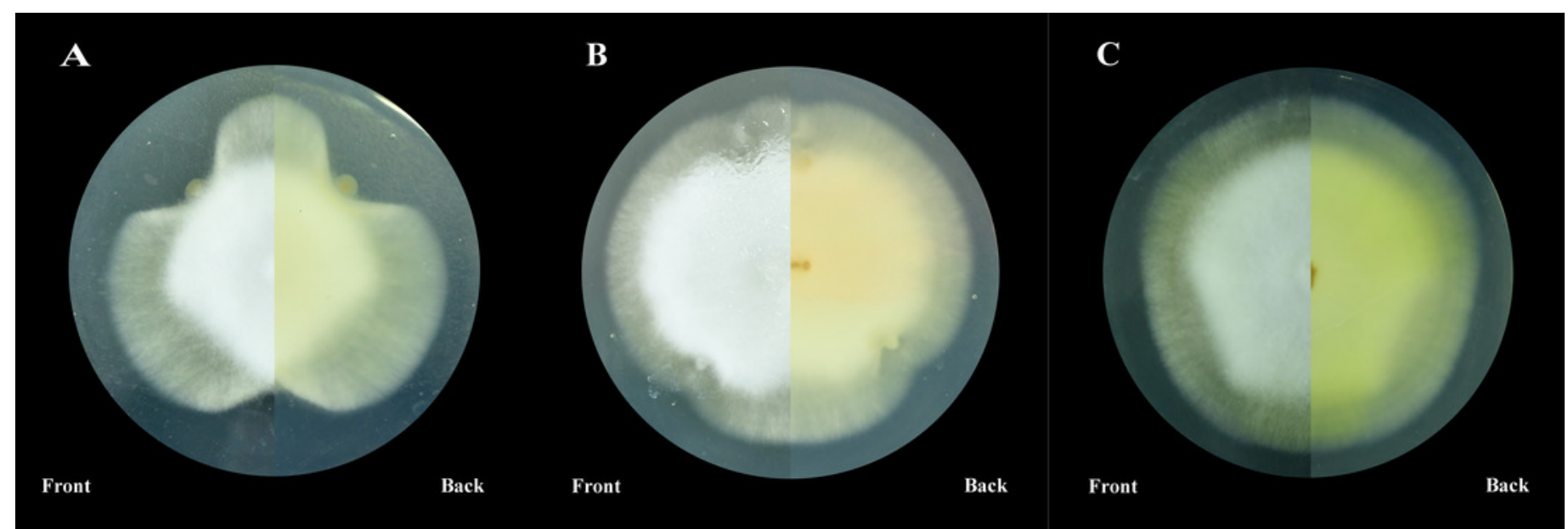


Figure 3

Effects of coculture of isolated bacterial strains with C. militaris.

(A) Dry weight of mycelium pellets. (B) Diameter of mycelium pellets. (C) Cordycepin content in dry weight of mycelium pellets. (D) Polysaccharide content in dry weight of mycelium pellets. **P $<0.01$.

A

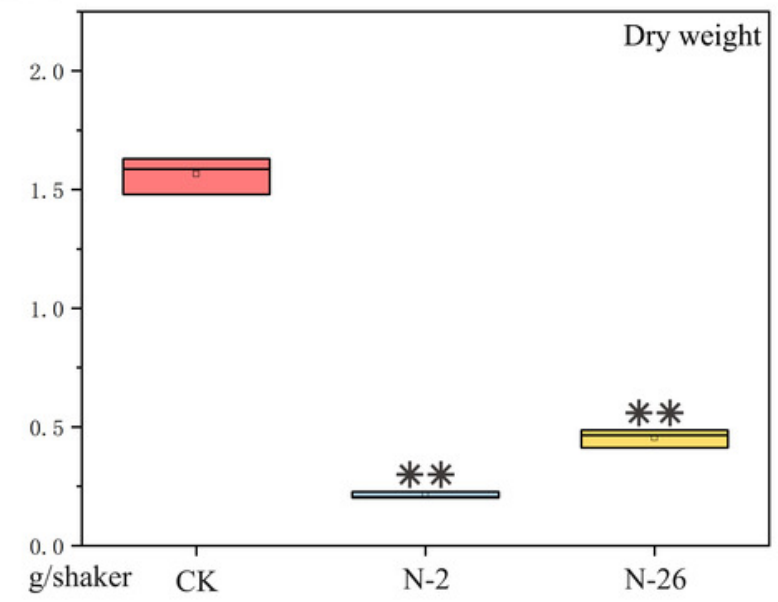

C

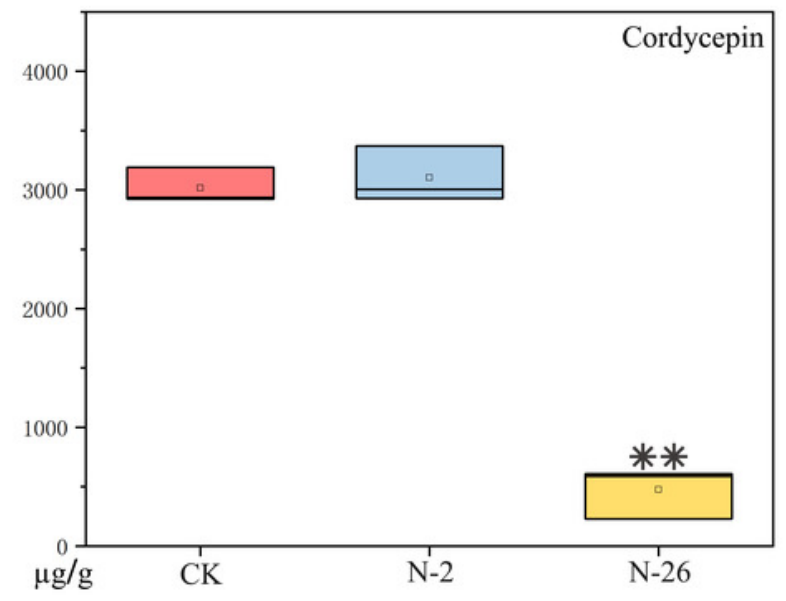

B

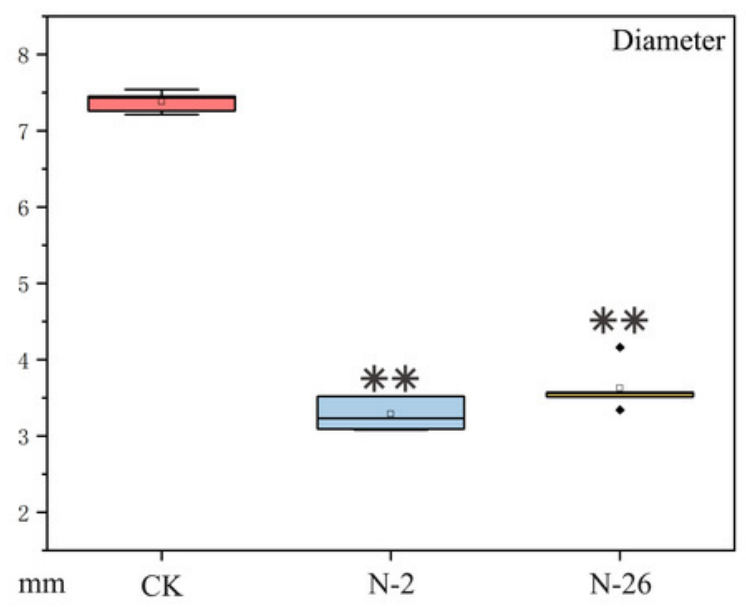

D

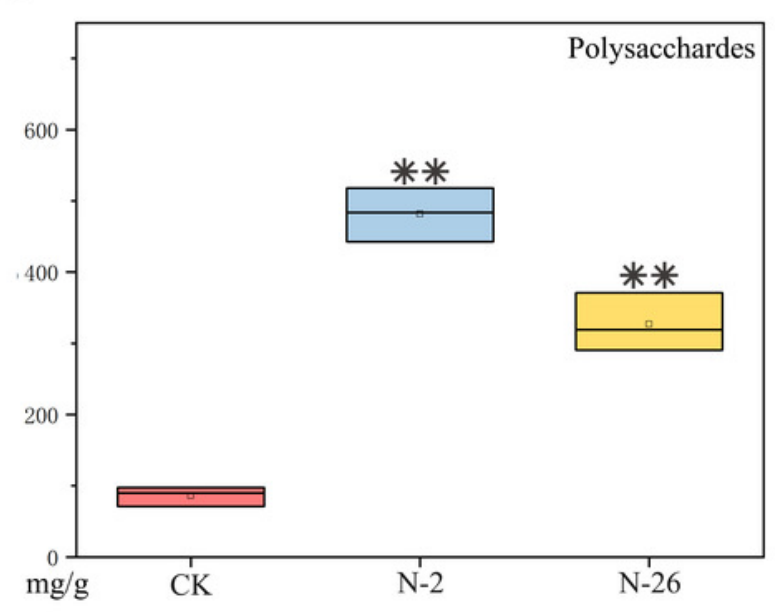




\section{Figure 4}

Taxonomic composition of the microbiome in sclerotia of Cordyceps militaris.

Circles from inside to outside represent the community composition of the bacteria at different classification levels (kingdom, phylum, class, order, family, and genus, respectively). The size of the fan segments represents the relative proportion of the annotation results of different bacterial OTUs. 


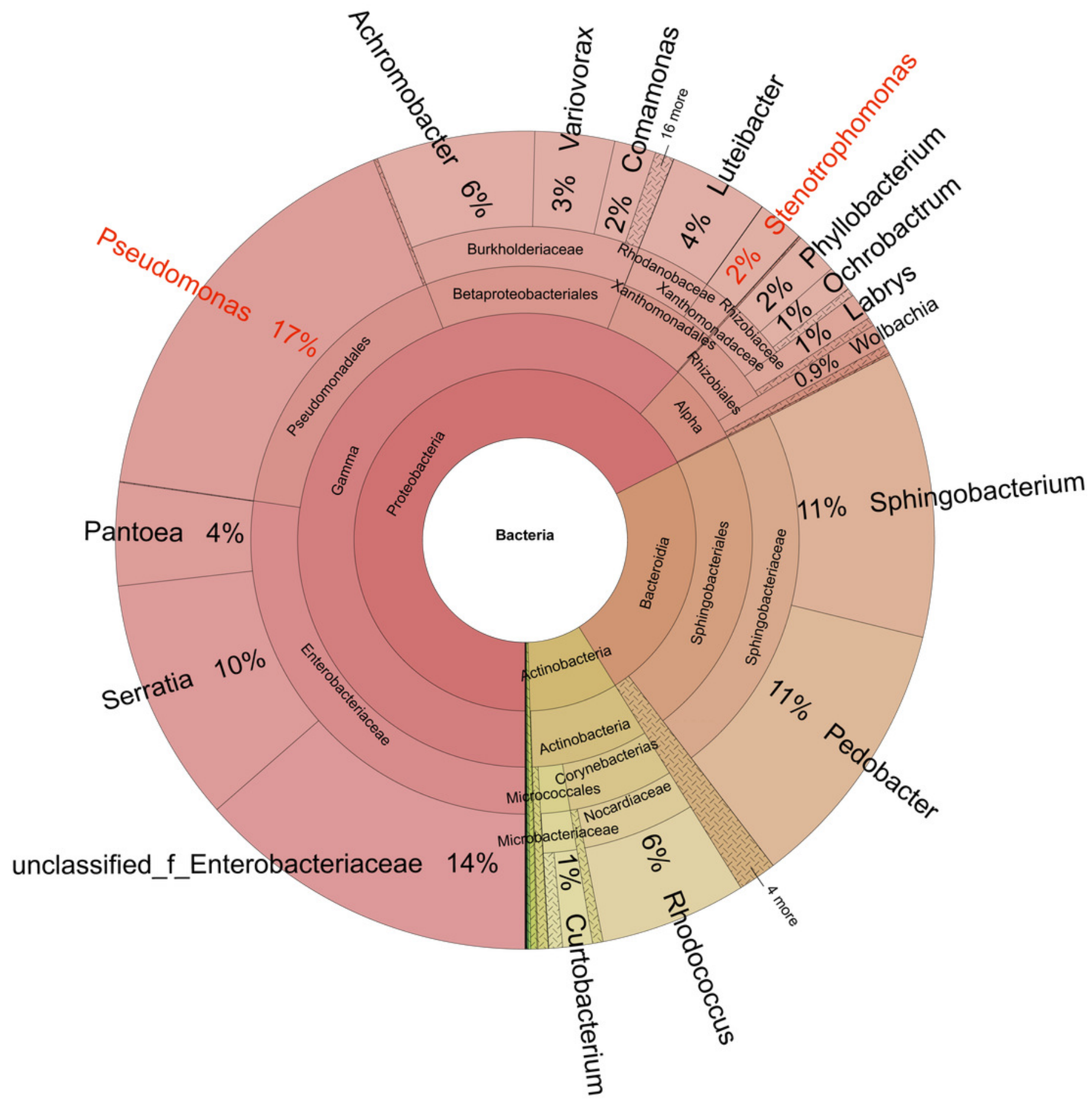


Figure 5

Functional prediction of the bacterial core microbiome in sclerotia of $C$. militaris using FAPROTAX.

Genera in red are the subject of this study. 

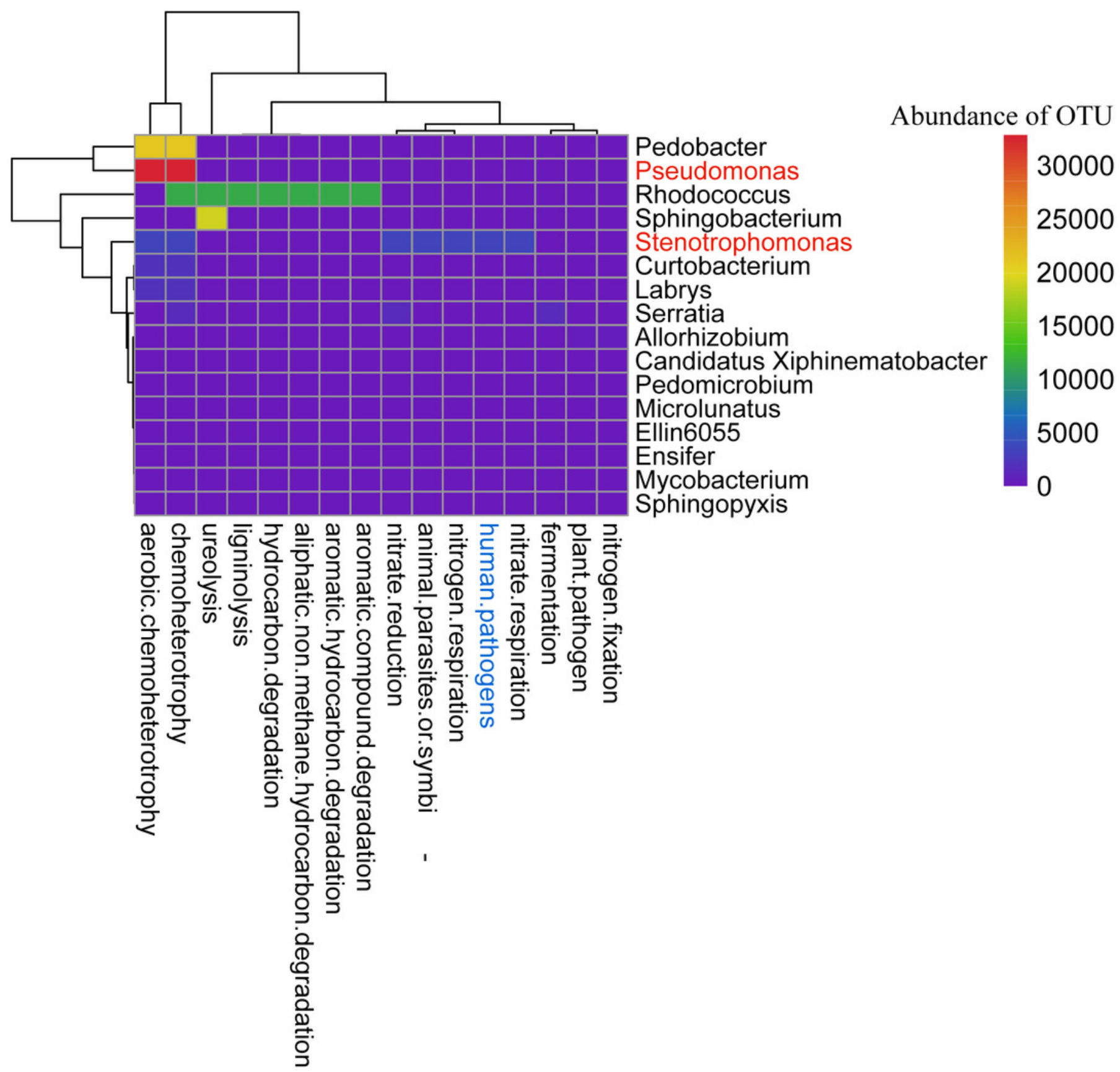


\section{Table 1 (on next page)}

Relative abundance and function prediction of bacterial composition in sclerotia of $C$. militaris isolates. 
1 Table 1 Relative abundance and function prediction of bacterial composition in sclerotia of $C$. militaris isolates.

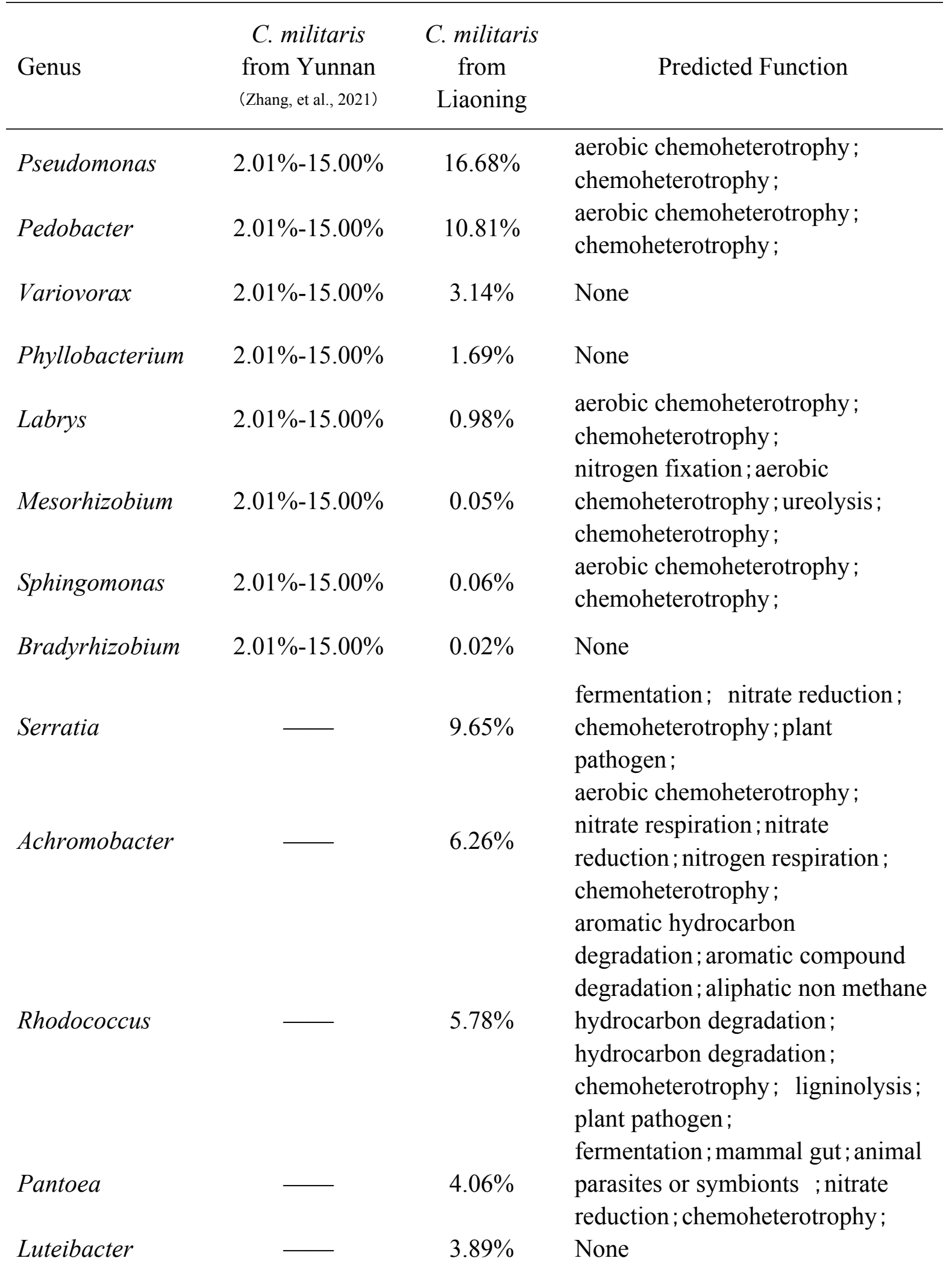




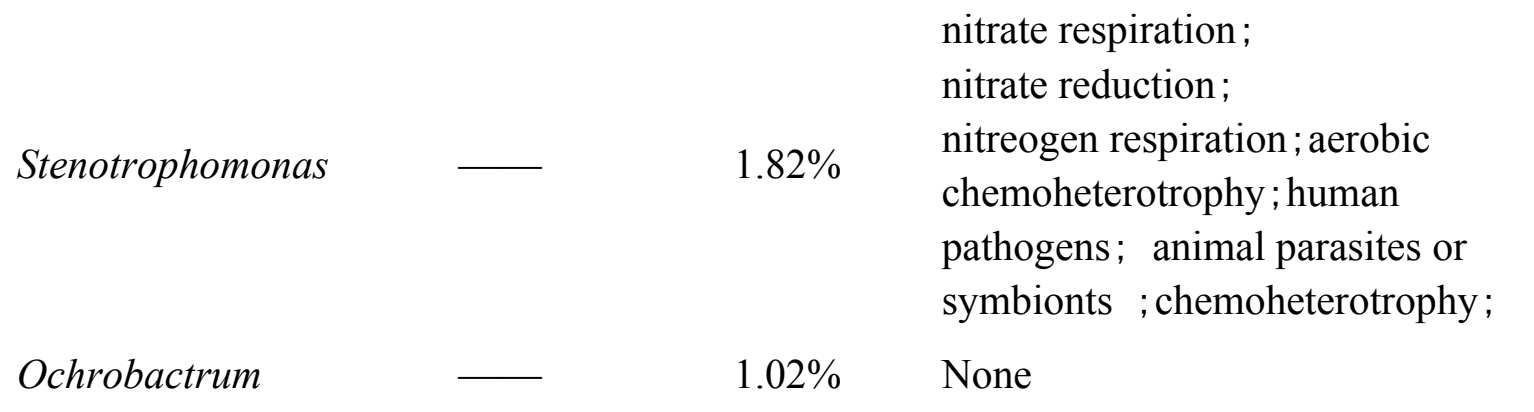

2 Note: "--" means not mentioned in the literature; "None" means that there was no result when a function was 3 predicted using FAPROTAX.

4 